EPJ manuscript No.

(will be inserted by the editor)

\title{
Linear hydrodynamics and viscoelasticity of nematic elastomers
}

\author{
E.M. Terentjev and M. Warner \\ Cavendish Laboratory, University of Cambridge, Madingley Road, Cambridge CB3 0HE, U.K.
}

October 29, 2018

\begin{abstract}
We develop a continuum theory of linear viscoelastic response in oriented monodomain nematic elastomers. The expression for dissipation function is analogous to the Leslie-Ericksen version of anisotropic nematic viscosity; we propose the relations between the anisotropic rubber moduli and new viscous coefficients. A new dimensionless number is introduced, which describes the relative magnitude of viscous and rubber-elastic torques. In an elastic medium with an independently mobile internal degree of freedom, the nematic director with its own relaxation dynamics, the model shows a dramatic decrease in the dynamic modulus in certain deformation geometries. The degree to which the storage modulus does not altogether drop to zero is shown to be both dependent on frequency and to be proportional to the semi-softness, the non-ideality of a nematic network. We consider the most interesting geometry for the implementation of the theory, calculating the dynamic response to an imposed simple shear and making predictions for effective moduli and (exceptionally high) loss factors.
\end{abstract}

PACS. 61.30.-v Liquid crystals. - 61.41.+e Polymers, elastomers and plastics - 83.50.Fc Linear viscoelasticity

\section{Introduction}

The equilibrium mechanical response of liquid crystalline elastomers can be soft or hard depending on the relation between the imposed strains and the nematic director, in particular, if the director is able to respond by rotating. With such unusual equilibrium elasticity one might expect dynamical response to be equally unusual. If the elastic forces are small, then the return to equilibrium is ' driven more weakly than in conventional systems. How does the dynamics of internal director rotation, and the corresponding time-dependent softening of rubber-elastic response, determine the dynamic mechanical response of a nematic rubber to a small amplitude oscillatory shear?

In a dynamic-mechanical study of Gallani et al. [1] a stress response to an imposed oscillating shear has been studied. Although a polydomain elastomer has been examined, the authors reached a conclusion that the response "is insensitive to the isotropic-nematic transition" and only obtained a non-trivial result in the smectic-A phase. Subsequent studies of aligned monodomain elastomers [2] also did not find any unusual effect in the nematic phase and went on to investigate the mechanical effects in the smectic-A phase. The elastic properties of smectic rubbers are very interesting on their own, with a number of spectacular effects even in equilibrium, see [3, 5, 6. 6. However, here we would like to address a more basic and physically clear case of nematic elastomers, that is, rubbery networks with an aligned uniaxial anisotropy of their polymer strands.
Some of the reasons that no exceptional effects were found in the nematic phase by [1,2] could be that (i) the nematic region for the materials studied was only $\sim 7^{\circ}$ and smectic pre-transitional effects were important, (ii) the authors aimed to plot the whole range of dynamic modulus, including very high glassy values at low temperatures, thus masking a subtle nematic region and (iii) they did not study low enough frequencies. More recently [7], it has been demonstrated, both theoretically and experimentally, that a dramatic reduction of storage modulus $G^{\prime}$ and the associated increase in the loss factor $\tan \delta$ should be expected and indeed found in monodomain nematic elastomers sheared in certain geometries (cf. Fig. 2). This effect allows one to directly probe the basic equilibrium properties of nematic rubbers and also access the new kinetic parameters - viscous coefficients and relaxation times.

In order to study the dynamics of mechanical response, one needs to model viscoelastic properties, that is, describe viscous dissipation in a system moving towards its equilibrium. At present, there is no microscopic model that would even approximately describe the dynamics of anisotropic rubbery networks. However, as in the continuum theory of liquid crystals, much progress can be made on a phenomenological level, using the symmetries of variables contributing to the physical effects.

The continuum dynamics of anisotropic fluids describes macroscopic phenomena on the level of coarse-graining analogous to that of the nematic Frank elastic free energy. By movements of a "liquid particle" one understands bod- 
Terentjev and Warner: Viscoelasticity of nematic elastomers

ily translations and also changes of local director orientation of a physically infinitesimal volume, generally of the correlation size $\xi_{\mathrm{N}} \sim 10 \mathrm{~nm}$, including many molecules in thermal equilibrium with the reservoir, and characterised by the local nematic tensor order parameter $Q_{\mathrm{ij}}(T)=$ $Q\left(n_{\mathrm{i}} n_{\mathrm{j}}-\frac{1}{3} \delta_{\mathrm{ij}}\right)$. Two physical fields describe the state of motion of nematic liquid: the local variation of director orientation $\delta \boldsymbol{n}(\boldsymbol{r}, t)=\boldsymbol{n}-\boldsymbol{n}_{0}$ with respect to the equilibrium $\boldsymbol{n}_{0}$ and the fluid velocity $\boldsymbol{v}(\boldsymbol{r}, t)$, the time derivative of the corresponding elastic displacement $\boldsymbol{u}(\boldsymbol{r}, t)$ in the description of elastic response. The original derivation of hydrodynamic equations for the nematic liquid, due to Ericksen and Leslie is presented in some detail in key monographs on liquid crystals, e.g. 87. Here we only discuss the matters relevant for the basic description of nematic elastomers, omitting many fine and subtle points of this complicated subject, e.g. [9]. We develop a formalism describing the linear viscoelasticity of an elastic medium with an independently relaxing director degree of freedom and make a connection between the (anisotropic) rubber-elastic and viscous coefficients in such a system. The nematic dynamics coupled to the underlying rubber elasticity leads to an expression for the entropy production density, the Rayleigh dissipation function in the LeslieEricksen form, determined by the fluxes: $\underline{\underline{\varepsilon}}$ - the symmetric strain rate and $\frac{d}{d t}(\boldsymbol{\Omega}-\boldsymbol{\omega})$ - the rate of relative rotation between the nematic director and the elastic matrix:

$$
\begin{aligned}
T \dot{s} & =A_{1}(\boldsymbol{n} \cdot \underline{\underline{\dot{\varepsilon}}} \cdot \boldsymbol{n})^{2}+2 A_{4}[\boldsymbol{n} \times \underline{\underline{\dot{\varepsilon}}} \times \boldsymbol{n}]^{2}+4 A_{5}([\boldsymbol{n} \times \underline{\underline{\dot{\varepsilon}}} \cdot \boldsymbol{n}])^{2} \\
& +\frac{1}{2} \gamma_{1}\left[\boldsymbol{n} \times \frac{d}{d t}(\boldsymbol{\Omega}-\boldsymbol{\omega})\right]^{2}+\gamma_{2} \boldsymbol{n} \cdot \underline{\underline{\dot{\varepsilon}}} \cdot\left[\boldsymbol{n} \times \frac{d}{d t}(\boldsymbol{\Omega}-\boldsymbol{\omega})\right]
\end{aligned}
$$

where the new linear viscous coefficients, $A_{\mathrm{i}}$, are linear combinations of the classical Leslie coefficients, which in elastomers can take extraordinarily high values [7] compared with simple nematics. $\gamma_{1}$ and $\gamma_{2}$ describe the same kind of losses involving the rotating director as they do in a conventional nematic liquid. We find a direct proportionality between the symmetry-related elastic and viscous constants, connected by the corresponding relaxation times for each deformation mode. One of the main results of our analysis is a new dimensionless number, which we call $\mathrm{Ne}=\eta v /(L \mu)$, where $\eta$ is a viscosity, $\mu$ is a rubber shear modulus and $L$ is a characteristic length. Ne determines the local balance between viscous and elastic torques in the material, in analogy with the Ericksen number $\operatorname{Er}$ [8].

An essential part of description developed in this paper is the separation of time scales. We argue, on the basis of comparison with available experiments and qualitative arguments, that the characteristic relaxation time of director motion is much greater than the time scale of mechanical relaxation in an ideal polymer network. Accordingly, we only consider the low-frequency limit of rubber viscoelastic response, reflected in essentially linear frequency dependence of complex modulus $G^{*}=G_{\mathrm{o}}+\mathrm{i} \omega \eta$. In contrast, the much slower director relaxation allows us to examine both its low- and high-frequency regimes.

This paper is organised as following. The next Section, continuing the introduction into the subject, briefly sketches the fundamentals of equilibrium nematic rub- ber elasticity, focusing on the linear continuum description rather than the full molecular theory valid to high deformations. Following this, in Section 3 we revisit the Leslie-Ericksen formulation of the continuum dissipation function $T \dot{s}$ and show that, as would be expected by symmetry, it is identical to the elastic energy density only with strains substituted by strain rates. We then discuss the fundamentals of nematic rubber viscoelasticity. In Section 4 the practical calculation of dynamic moduli $G^{*}(\omega)$ is presented for three principal shear geometries, as an example of general theory implementation. The results indicate which combinations of elastic and viscous coefficients are relevant for the response and, therefore, directly measurable by experiment. We then conclude by discussing the role of soft elasticity in slowing the mechanical relaxation and make contact with new experimental data.

\section{Nematic rubber elasticity}

Equilibrium elastic properties of monodomain nematic rubbers are well-studied, both theoretically and experimentally and are described at some length in review articles [10,11]. A full molecular theory of ideal nematic networks gives the elastic free energy density

$$
F=\frac{1}{2} c_{\mathrm{x}} k_{\mathrm{B}} T \operatorname{Tr}\left(\underline{\underline{\boldsymbol{\lambda}}}^{T} \cdot \underline{\underline{\ell}}_{\theta}^{-1} \cdot \underline{\underline{\boldsymbol{\lambda}}} \cdot \underline{\underline{\boldsymbol{\ell}}}_{0}\right)+\frac{1}{2} \widetilde{B}(\operatorname{Det}[\underline{\underline{\boldsymbol{\lambda}}}]-1)^{2}
$$

where $\underline{\underline{\lambda}}$ is a Cauchy strain tensor, $\lambda_{\mathrm{ik}}=\partial R_{\mathrm{i}} / \partial R_{\mathrm{k}}^{0}$. In a nematic elastomer network, $\underline{\underline{\ell}}$ are the uniaxial matrices of chain step-lengths before $(0)$ and after the director $\boldsymbol{n}$ has rotated by a certain angle $\theta$ during the deformation: $\ell_{i j}=\ell_{\perp} \delta_{i j}+\left[\ell_{\|}-\ell_{\perp}\right] n_{i} n_{j}$. The last term in (1), the bulk-modulus contribution independent of the configurational entropy of polymer chains, is determined by molecular forces resisting the compression of a molecular liquid, $\widetilde{B} \sim 10^{9} \mathrm{~J} / \mathrm{m}^{3}$, much greater than the typical value of rubber modulus $\mu \simeq c_{\mathrm{x}} k_{\mathrm{B}} T$, with $c_{\mathrm{x}}$ the effective crosslinking density, giving $\mu \sim 10^{5} \mathrm{~J} / \mathrm{m}^{3}$. This large bulk energy penalty constrains the value of the strain determinant, $\operatorname{Det}[\underline{\underline{\lambda}}] \approx 1$ (which, in other words, means that the material is physically incompressible when subjected to all strains except hydrostatic compressions and dilations). Eq. (1) is only limited by the Gaussian network assumption and is valid up to very large strains where mechanical softness, rotational instabilities and optical effects occur well beyond the limits of continuum theory.

Olmsted [12], in studying soft elasticity - shape changes in nematic elastomers without energy cost, has derived the small-deformation continuum limit of the full expression (11), when $\lambda_{\mathrm{ij}}=\delta_{\mathrm{ij}}+\partial_{\mathrm{i}} u_{\mathrm{j}}$ and $|\nabla \boldsymbol{u}| \ll 1$. We shall use a slightly different notation, more suitable for the linear-elastic description because it better complies with the standard textbook formalism of uniaxial elasticity, e.g. [13]. The small-deformation limit of (11) is

$$
\begin{aligned}
F & =C_{1}(\boldsymbol{n} \cdot \underline{\underline{\tilde{\varepsilon}}} \cdot \boldsymbol{n})^{2}+2 C_{2} \operatorname{Tr}[\underline{\underline{\varepsilon}}](\boldsymbol{n} \cdot \underline{\underline{\tilde{\varepsilon}}} \cdot \boldsymbol{n})+C_{3}(\operatorname{Tr}[\underline{\underline{\varepsilon}}])^{2} \\
& +2 C_{4}[\boldsymbol{n} \times \underline{\underline{\tilde{\varepsilon}}} \times \boldsymbol{n}]^{2}+4 C_{5}([\boldsymbol{n} \times \underline{\underline{\tilde{\varepsilon}}} \cdot \boldsymbol{n}])^{2} \\
& +\frac{1}{2} D_{1}[\boldsymbol{n} \times(\boldsymbol{\Omega}-\boldsymbol{\omega})]^{2}+D_{2} \boldsymbol{n} \cdot \underline{\underline{\tilde{\varepsilon}}} \cdot[\boldsymbol{n} \times(\boldsymbol{\Omega}-\boldsymbol{\omega})]
\end{aligned}
$$


where $\boldsymbol{n}$ is the undistorted nematic director. $\tilde{\varepsilon}_{\mathrm{ik}}=\varepsilon_{\mathrm{ik}}-$ $\frac{1}{3} \operatorname{Tr}[\underline{\underline{\varepsilon}}] \delta_{\mathrm{ik}}$ is the traceless part of linear symmetric strain $\varepsilon_{\mathrm{ik}}=\frac{1}{2}\left(\partial_{\mathrm{k}} u_{\mathrm{i}}+\partial_{\mathrm{i}} u_{\mathrm{k}}\right)$, which is the only variable of classical continuum elasticity [13]. In a system with an internal orientational degree of freedom, the nematic director with its own relaxation and dynamics, the antisymmetric part of strain expressed by the local rotation vector $\boldsymbol{\Omega}=\frac{1}{2} \operatorname{curl} \boldsymbol{u}$ may contribute to the physical properties. Analogously, the small vector $\boldsymbol{\omega}$ is a convenient measure of director rotations, $\boldsymbol{\omega}=[\boldsymbol{n} \times \delta \boldsymbol{n}]$. In fact, it is the relative rotation, the difference $\boldsymbol{n} \times(\boldsymbol{\Omega}-\boldsymbol{\omega})$, that causes the elastic response and a number of effects unique to nematic elastomers [14].

One expects that in a rubber or dense polymer melt the bulk modulus $C_{3}$ is very large, $C_{3} \sim \widetilde{B}$. We, therefore, shall only consider deformations with no bulk compression: $\operatorname{Tr}[\underline{\underline{\varepsilon}}]=0$. In general, all other constants in the expression (2) are of the same order of magnitude, similar to the rubber modulus $\mu$. The molecular model of ideal nematic elastomer [11] gives specific forms for these constants:

$$
\begin{aligned}
& C_{1}=2 C_{4}=c_{\mathrm{x}} k_{\mathrm{B}} T, \quad C_{5}=\frac{1}{8} c_{\mathrm{x}} k_{\mathrm{B}} T \frac{(r+1)^{2}}{r}, \\
& D_{1}=c_{\mathrm{x}} k_{\mathrm{B}} T \frac{(r-1)^{2}}{r}, \quad D_{2}=c_{\mathrm{x}} k_{\mathrm{B}} T \frac{1-r^{2}}{r},
\end{aligned}
$$

in which case the condition for ideal soft elasticity holds,

$$
C_{5}^{R}=C_{5}-\frac{D_{2}^{2}}{8 D_{1}}=0 .
$$

Model expressions for elastic constants (3) depend, apart from the universal rubber-elastic energy scale $\mu$, on a single parameter $r$. In the molecular model (11) of ideal nematic polymer networks one finds that $r=\ell_{\|} / \ell_{\perp}$, the ratio of the principal step lengths of the anisotropic polymer backbone (or equivalently $r=\left(R_{\|} / R_{\perp}\right)^{2}$ in terms of the principal values of gyration radii tensor). In non-ideal elastomers, this parameter is more complex, determined by a number of other factors, for instance when there are fluctuations in composition, see the Appendix. Nevertheless, it has to be a function of nematic order parameter $Q(T)$, satisfying a linear limit $r \approx 1+\beta Q$, at least at small $Q$. In the isotropic phase, at $Q=0$ and $r=1$, the elastic constants (3) become, as expected: $C_{1}=2 C_{4}=2 C_{5}=\mu$, $D_{1}=D_{2}=0$ and the elastic energy (2) reduces to a standard Lamé expression. See the Appendix for a discussion of non-ideality, that is where $C_{5}^{R} \neq 0$.

In an incompressible material, all deformations are essentially shears, albeit sometimes seen in a rotated frame. Figure 1 shows the character of these shears in the eq. (2) and their corresponding elastic constants. $C_{1}$ "lives" along the director, $C_{4}$ in the plane perpendicular to $\boldsymbol{n}_{0}$ in which properties are isotropic $\left(\varepsilon_{\mathrm{kk}}, \varepsilon_{\mathrm{mm}}\right.$ and $\varepsilon_{\mathrm{mk}}$ are of the same status since the solid is uniaxial). Most interesting are $C_{5}$, involving shears such as $\varepsilon_{\mathrm{nk}}$, which span directions parallel and perpendicular to the initial director and which can induce it to rotate; $D_{1}$ which resists director rotations with respect to the solid matrix; and $D_{2}$ where the rotation is coupled to the symmetric shears also involved in $C_{5}$. The

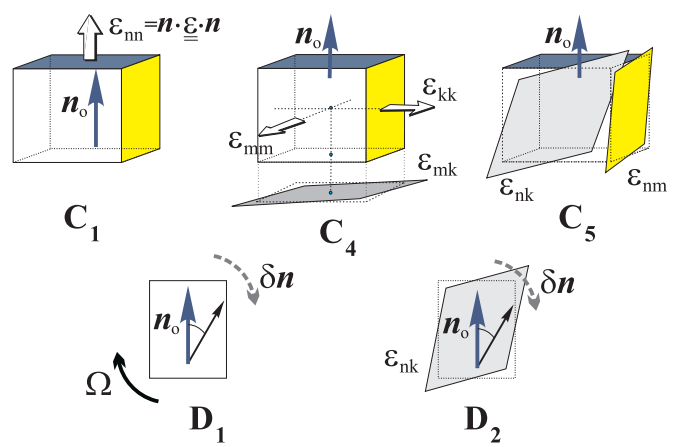

Fig. 1. The symmetries of the shears and rotations possible in a uniaxial solid at constant volume and with an internal orientational degree of freedom. The modes are labeled with the appropriate elastic constant and symmetric shear or rotation. $C_{4}$ also shows a section perpendicular to the director to illustrate shears $\varepsilon_{\mathrm{mk}}$ not involving $\boldsymbol{n}$ (the directions $\boldsymbol{m}$ and $\boldsymbol{k}$ are perpendicular to $\boldsymbol{n})$. In the two coupling terms, $D_{1}$ and $D_{2}$, the small director rotation $|\boldsymbol{\omega}| \approx|\delta \boldsymbol{n}|$.

inter-relation between these three processes is what gives rise to the effect of soft elasticity - here shape change of the type $\varepsilon_{\text {nk }}$ without energy cost. If the director is allowed to react ideally and is regarded as a slave variable, then the renormalised resistance to deformations $\varepsilon_{\mathrm{nk}}$, that is $C_{5}^{R}$, vanishes as we see in eq. (四). We shall see, in Section 4 for simple shear geometries, the mechanism by which this is achieved - at least at harmonic order (since these simple geometries do not allow the full shear and extensional freedom required for softness at all amplitudes).

\section{Nematohydrodynamics of elastic solids}

When nematic elastomers are strained, there is director rotation along with stresses - the latter yielding both body forces and torques. At finite strain and rotational rates there will be stresses of both elastic and viscous origin, the latter being conceptually parallel to those arising in classical liquid nematics. We shall accordingly review classical nematohydrodynamics, ignoring the subtleties that reside at the heart of the subject. There are important differences with liquids - if torques are induced by flow, they will be balanced in solids, on length scales longer than the nematic penetration depth, by torques generated by the matrix, that is, by $D_{1}$ and $D_{2}$ elastic terms that involve the anti-symmetric part of the strain $\underline{\underline{\lambda}}$. The issue of possible director gradients and corresponding Frank elasticity in nematic elastomers has been extensively discussed in the literature [10,11, 15]. It is known that, unless there are special reasons for a director singularity (such as in disclinations or narrow domain walls), Frank elastic effects play a minor role in the free energy balance and can be neglected. Instead, the rubber-elastic matrix generates the torques, which shape the final character of the dynamics. A new dimensionless number will be introduced to replace the Ericksen number describing a torque balance in ordinary nematics. There is another important difference - in 
liquids, as flow proceeds, the strain and the director rotation can increase without limit, whereas in solids both are constrained (and are coupled to each other) by the matrix. Elastomers are capable of huge distortions but we shall limit ourselves to linear theory. Nematohydrodynamics in the elastically highly non-linear regime is a subject of another study.

\subsection{Leslie-Ericksen formulation of nematic viscosity}

The equation of motion can be written in the usual form of local balance of forces: in vector components,

$$
\frac{\partial}{\partial t}\left(\rho v_{\mathrm{i}}\right)=-\frac{\partial \Pi_{\mathrm{ki}}}{\partial x_{\mathrm{k}}}
$$

where $\rho$ is the density and $\Pi_{\mathrm{ki}}$ the tensor of momentum flux density, $\Pi_{\mathrm{ki}}=\rho v_{\mathrm{k}} v_{\mathrm{i}}-\sigma_{\mathrm{ki}}+P \delta_{\mathrm{ki}}$ with $P$ the local pressure and $\sigma_{\mathrm{ki}}$ the stress tensor. In an isotropic Newtonian liquid $\sigma_{\mathrm{ki}}$ is proportional to the symmetric strain rate, $A_{\mathrm{ki}}=\frac{1}{2}\left(\partial_{\mathrm{k}} v_{\mathrm{i}}+\partial_{\mathrm{i}} v_{\mathrm{k}}\right) \equiv \dot{\varepsilon}_{\mathrm{ki}}$. In uniaxial nematic liquid crystals, the stress tensor depends not only on the fluid velocity gradients, but also on the components and the gradients of the local nematic order $Q_{\mathrm{ki}}$. Because rotations and corresponding torques are involved if the director changes differently from the local fluid rotation, the stress tensor is no longer symmetric and also depends on the relative rotation combinations $\boldsymbol{N}=\frac{d}{d t} \delta \boldsymbol{n}+\frac{1}{2}[\boldsymbol{n} \times \operatorname{curl} \boldsymbol{v}]$ [8], which is nothing but a time derivative of the relative-rotation combination, that is $\boldsymbol{N}=\left[\boldsymbol{n} \times \frac{d}{d t}(\boldsymbol{\Omega}-\boldsymbol{\omega})\right]$, cf. equation (2). As a result, Leslie writes the viscous stress tensor

$$
\begin{aligned}
\sigma_{\mathrm{ij}}= & \alpha_{1} n_{\mathrm{i}} n_{\mathrm{j}} A_{\mathrm{km}} n_{\mathrm{k}} n_{\mathrm{m}}+\alpha_{2} n_{\mathrm{i}} N_{\mathrm{j}}+\alpha_{3} n_{\mathrm{j}} N_{\mathrm{i}} \\
& +\alpha_{4} A_{\mathrm{ij}}+\alpha_{5} n_{\mathrm{i}} n_{\mathrm{k}} A_{\mathrm{kj}}+\alpha_{6} n_{\mathrm{j}} n_{\mathrm{k}} A_{\mathrm{ki}}
\end{aligned}
$$

(assuming incompressible fluid, $A_{\mathrm{kk}}=\operatorname{div} \boldsymbol{v}=0$ ). Its antisymmetric part contributes to the local torque $\boldsymbol{\Gamma}=[\boldsymbol{n} \times \boldsymbol{h}]$ (cf. [8]) where $\boldsymbol{h}$ is the molecular field. $\boldsymbol{h}$ derives also in part from Frank and external field terms. These contributions are balanced by the anti-symmetric terms deriving from viscous flow processes:

$$
h_{\mathrm{i}}=\frac{\partial}{\partial x_{\mathrm{k}}}\left(\frac{\delta F}{\delta\left[\nabla_{\mathrm{k}} n_{\mathrm{i}}\right]}\right)-\frac{\partial F}{\partial n_{\mathrm{i}}}=\gamma_{1} N_{\mathrm{i}}+\gamma_{2} n_{\mathrm{k}} A_{\mathrm{ki}}
$$

with $\gamma_{1}=\alpha_{3}-\alpha_{2}$ and $\gamma_{2}=\alpha_{3}+\alpha_{2}=\alpha_{6}-\alpha_{5}$ (the Parodi relation, a representation of the Onsager principle of kinetic coefficient symmetry). Here the viscous coefficients $\alpha_{1}, \ldots, \alpha_{6}$ all depend on the magnitude of nematic order parameter $Q(T)$. One can show that in an ordinary nematic liquid crystal near the weak first order transition, as $Q \rightarrow 0$, they should behave as 16

$$
\begin{array}{r}
\alpha_{1} \propto Q^{2},\left(\alpha_{2}, \alpha_{3}, \alpha_{5}, \alpha_{6}\right) \propto Q \\
\text { also } \gamma_{1} \propto Q^{2} \text { and } \gamma_{2} \propto Q .
\end{array}
$$

Thus, in the isotropic phase, only one of the Leslie coefficients survives: $\alpha_{4} \rightarrow 2 \eta$. Molecular theory [17, 18] also shows that in a typical nematic liquid of rod-like molecules these coefficients may have very different magnitude and even sign: $\alpha_{2}, \alpha_{3}, \alpha_{6}$ - negative, $\alpha_{4}, \alpha_{5}$ - positive; $\left(\left|\alpha_{2}\right| \sim\right.$ $\left.\alpha_{5}\right) \gg\left|\alpha_{3}\right|,\left|\alpha_{6}\right| ; \alpha_{1}$ is generally small and may be positive or negative in different materials; far from the nematic transition (at $Q \rightarrow 1$ ) the "isotropic coefficient" $\alpha_{4} \sim\left|\alpha_{2}\right| \sim \alpha_{5}$. De Gennes assembles the experimental values for Leslie coefficients in MBBA at $25^{\circ} \mathrm{C}$ [8], while the monograph by de Jeu [19 provides the values for another classical nematic, PAA at $122^{\circ} \mathrm{C}$ : in $10^{-3} \mathrm{~Pa} . \mathrm{s}$

$\begin{array}{rcccccccc} & \alpha_{1} & \alpha_{2} & \alpha_{3} & \alpha_{4} & \alpha_{5} & \alpha_{6} & \gamma_{1} & \gamma_{2} \\ \text { MBBA : } & 6.5 & -77.5 & -1.2 & 83.2 & 46.3 & -34.4 & 76.3 & -78.7 \\ \text { PAA : } & 4 & -6.9 & -0.2 & 6.8 & 5 & -2 & 6.8 & -7.1\end{array}$

\subsection{Nematic elastomers}

We now develop Leslie-Erickson theory for solid nematics by a direct analogy. The nematic dynamics is coupled to the underlying anisotropic elasticity described in its linear limit by eq. (2) and sketched in Fig. 1. It is thus natural to develop the dynamics using the same symmetry-grouping of terms as in (2), rather than as in (6) as is usual for classical nematics.

Two differential equations, (5) with the viscous stress given by eqs. (6), and (7), form the complete set describing the linear viscous effects in the nematic fluid. Neglecting the effects of heat convection, the total energy dissipation (the entropy production) in such anisotropic medium is expressed by the volume integral of the conjugate forces and fluxes:

$$
\begin{aligned}
T \dot{S} & =\int d V\left(\sigma_{\mathrm{ij}} A_{\mathrm{ij}}+(\boldsymbol{h} \cdot \dot{\boldsymbol{n}})-(\boldsymbol{\Gamma} \cdot \boldsymbol{\Omega})\right) \\
& \equiv \int d V\left(\sigma_{\mathrm{ij}} \dot{\varepsilon}_{\mathrm{ij}}+(\boldsymbol{h} \cdot \boldsymbol{N})\right)
\end{aligned}
$$

We re-write the density of dissipation function in a form matching the elastic energy density (2):

$$
\begin{aligned}
T \dot{s}= & A_{1}(\boldsymbol{n} \cdot \underline{\underline{\dot{\varepsilon}}} \cdot \boldsymbol{n})^{2}+2 A_{4}[\boldsymbol{n} \times \underline{\underline{\dot{\varepsilon}}} \times \boldsymbol{n}]^{2}+4 A_{5}([\boldsymbol{n} \times \underline{\underline{\dot{\varepsilon}}} \cdot \boldsymbol{n}])^{2} \\
& +\frac{1}{2} \gamma_{1} \boldsymbol{N}^{2}+\gamma_{2} \boldsymbol{n} \cdot \underline{\underline{\dot{\varepsilon}}} \cdot \boldsymbol{N}
\end{aligned}
$$

in the fully incompressible case. Thus, by differentiation of (10) respectively by the symmetric strain rate $\varepsilon_{i j}$ and by $N_{i}$, one obtains a representation of the symmetric viscous stress tensor and the nematic molecular field, contributing to the local torque, analogous to the parallel expressions (6) and (7) for simple nematics:

$$
\begin{aligned}
\sigma_{\mathrm{ij}}^{s} & =2 A_{1}(\boldsymbol{n} \cdot \underline{\underline{\dot{\varepsilon}}} \cdot \boldsymbol{n}) n_{i} n_{j}+4 A_{4}[\boldsymbol{n} \times(\boldsymbol{n} \times \underline{\underline{\dot{\varepsilon}}} \times \boldsymbol{n}) \times \boldsymbol{n}]_{i j} \\
& +4 A_{5}\left([(\boldsymbol{n} \times \underline{\underline{\dot{\varepsilon}}} \cdot \boldsymbol{n}) \times \boldsymbol{n}]_{i} n_{j}+[(\boldsymbol{n} \times \underline{\underline{\dot{\varepsilon}}} \cdot \boldsymbol{n}) \times \boldsymbol{n}]_{j} n_{i}\right) \\
& +\frac{1}{2} \gamma_{2}\left(n_{i} N_{j}+N_{i} n_{j}\right) ; \\
h_{\mathrm{i}} & =\gamma_{1} N_{i}+\gamma_{2} n_{j} \dot{\varepsilon}_{i j},
\end{aligned}
$$

where $\sigma_{\mathrm{ij}}^{s}$ is the symmetric part of an expression like (6) which emerges naturally since we express $T \dot{s}$ in terms of symmetric and anti-symmetric variables separately. Here 
the constants are linear combinations of classical Leslie coefficients

$$
\begin{aligned}
& A_{1}=\frac{1}{2}\left(\alpha_{1}+\alpha_{4}+\alpha_{5}+\alpha_{6}\right), \\
& A_{4}=\frac{1}{4} \alpha_{4}, \\
& A_{5}=\frac{1}{8}\left(2 \alpha_{4}+\alpha_{5}+\alpha_{6}\right) .
\end{aligned}
$$

In the isotropic limit, one finds $A_{1}=2 A_{4}=2 A_{5} \rightarrow \eta$, reminiscent of the isotropic Lamé limit of the corresponding elastic constants.

\subsection{Relaxation times}

The direct correspondence between the elastic constants of the energy density $F$, eqs. (2), and the viscous coefficients of the dissipation function $\dot{T} \dot{s}$, eq. (10), gives:

$$
A_{\mathrm{i}} \rightarrow C_{\mathrm{i}} ; \gamma_{1} \rightarrow D_{1} \text { and } \gamma_{2} \rightarrow D_{2}
$$

Of course, this is not a surprise since the symmetry of an equilibrium elastic deformation and of a viscous flow in a uniaxial continuum is the same. Equally, there is a direct correspondence in the dependence of the various coefficients on nematic order parameter $Q$, see eq. (8) for viscosities and eq. (3) for elastic constants. We can equivalently, therefore, represent the viscous coefficients of a nematic elastomer by products of the corresponding rubberelastic constant and an appropriate relaxation time, that is,

$$
A_{\mathrm{i}}=C_{\mathrm{i}} \tau_{\mathrm{R}} ; \gamma_{1}=D_{1} \tau_{1} ; \gamma_{2}=D_{2} \tau_{2}
$$

In principle, the relaxation times for the various $A_{i}$ will be different, and different from the $\tau_{i}$. However, one expects a significant separation between the two groups of characteristic time scales, that is between the $\tau_{\mathrm{R}} \mathrm{S}$ and the $\tau_{i} \mathrm{~s}$. The director rotation time, $\tau_{1}=\gamma_{1} / D_{1}$, has been used in a simplified, single-viscosity analysis [7], and then estimated experimentally as $\tau_{1} \sim 10^{-1}-10^{-2}$ S. In contrast, the characteristic time of rubber relaxation $\tau_{\mathrm{R}}$ is expected to be much shorter. A recent statistical theory explicitly calculated this time from the analysis of network strand relaxation within a tube model [20], showing this is of order of Rouse time. One could also confirm this by a following qualitative observation: The linear viscous stress (11) and the analogous corresponding expression for the elastic stress could be combined, producing the effective frequency-dependent moduli in a form $\left(C_{\mathrm{i}}+\mathrm{i} \omega A_{\mathrm{i}}\right)$. This is the low-frequency limit of a general complex modulus $G^{*}(\omega)$, showing the rubber plateau modulus $G^{\prime}=C_{\mathrm{i}}$ and the initial rise in the loss modulus with frequency, describing a viscous flow. In the classical picture of polymer dynamics, e.g. 21, the next characteristic regime is at a Rouse frequency, when the signal cannot propagate along the polymer chain length and mechanical response is provided by individual segments, thus causing a climb of $G^{*}(\omega)$ towards the glass plateau. Therefore, the characteristic time scale $\tau_{\mathrm{R}}$ in the estimation relations (13) is of the order of the Rouse time, $\tau_{\mathrm{R}} \sim 10^{-4}-10^{-6} \mathrm{~s}$.

Could there be viscous softness analogously to the elastic softness of ideal elastomers, for instance in eq. (4) ? Two related arguments suggest that this is not possible. The viscous combination $A_{5}-\gamma_{2}^{2} / 8 \gamma_{1}$, analogous to the renormalised elastic constant $C_{5}^{R}$, can be rewritten with the benefit of timescales eq. (13) as:

$$
A_{5}-\gamma_{2}^{2} / 8 \gamma_{1}=\tau_{\mathrm{R}}\left(C_{5}-\frac{\tau_{2}^{2}}{\tau_{\mathrm{R}} \tau_{1}} \frac{D_{2}^{2}}{8 D_{1}}\right)
$$

Even if we took the rotational relaxation times equal, $\tau_{1}=\tau_{2}$, the translational relaxation time $\tau_{\mathrm{R}}$ would appear to upset the possibility of renormalising (14) to zero, even if we had $C_{5}^{R} \rightarrow 0$. Elastic softness arises because an anisotropic distribution of chains can be rotated undistorted and thus at constant entropy. However, individual chains will be distorted and there must reasonably be dissipation associated with their flow relative to the matrix. Thus elastically soft distortions should have associated dissipation, except perhaps accidentally if rates of $\gamma_{1}, \gamma_{2}$ and $A_{5}$ relaxations compensate to make (14) vanish.

\subsection{Symmetries and order parameter}

The ability to neglect complicated inertial effects in fluid dynamics is controlled by the small magnitude of Reynolds number, $R e=\rho v L / \eta \ll 1$, with $L$ the characteristic length and $\eta$ the typical viscosity. In nematic liquids, another dimensional number is introduced to characterise the relative magnitude of hydrodynamic and Frank elastic torques contributing to eq. (7), where the left hand side is $\sim K \nabla^{2} n$, while the right hand side is $\sim \gamma \nabla v$, with the rotational viscosity $\gamma \sim \eta$. Small Ericksen number $E r=\eta v L / K \ll 1$ means that the director orientation is mostly controlled by equilibrium elastic free energy, while at $E r \gg 1$ the director generally follows the local orientation provided by the flow. In elastomers we are concerned with the balance between flow-induced torques, again scaling as $\sim \eta \nabla v$, and those of the rubbery matrix, expressed by $\partial F / \partial \theta \sim D_{1} \theta$. This yields a new dimensionless group characterised by the number $\mathrm{Ne}=\eta v /\left(L D_{1}\right)$. Note that the assumed domination of rubber-elastic over Frank effects essentially means that $D_{1} \gg K / L^{2}$, that is, $\mathrm{Ne} \ll E r$. Our analysis of an example of simple shear deformation, in the next Section, spans the full range of small to large $\mathrm{Ne}$, which we shall define more precisely for that geometry.

We have seen in Section 2, in the discussion of eqs. (3) and chain-anisotropy parameter $r$, how the anisotropic rubber moduli depend on the underlying nematic order parameter $Q$, usually a function of temperature or solvent concentration. In the isotropic phase, at $Q=0$ and $r=1$, the elastic constants return to the classical Lamé values $C_{1}=2 C_{4}=2 C_{5}=\mu, D_{1}=D_{2}=0$. The coupling constant $D_{2}$ depends on the linear power of $Q$. As a result, when there is anisotropy, $Q \neq 0$ and $r \neq 1$, the sign of the elastic constant $D_{2}$ depends on whether $r>1$ (prolate order, $D_{2}<0$ ) or $r<1$ (oblate order, $\left.D_{2}>0\right)$. The sign of director rotation relative to the matrix, $\boldsymbol{\omega}-\boldsymbol{\Omega}$, varies accordingly to whether chains are prolate (they align with the extension direction associated 
with the shear, see Fig. 1 for $D_{2}$ ) or oblate (alignment along the compression axis). In any event, a term such as $D_{2} \varepsilon_{\mathrm{nk}} \omega$, bilinear in $\varepsilon_{\mathrm{nk}} \omega$, always reduces the elastic energy, irrespective of the sign of $D_{2}$, and indeed this is the actual mechanism whereby a shape change can ideally be achieved with no energy cost [12, 22]. There is an appealing analogy between this behaviour and the Leslie coefficient $\gamma_{2}$ of discotic nematics. It is known [24, 25] that the different (disk- instead of rod-like) molecular shape leads to the opposite sign of $\gamma_{2}$, with the according consequences for the flow alignment properties.

Ideal rubber-elastic expressions (3) suggest that the coupling constant $D_{1}$ is proportional to $(r-1)^{2} \sim Q^{2}$. Continuing the analogy with liquid nematic viscosity, we should recall the estimates of Imura and Okano [16], but also a following qualitative consideration. The rotational viscosity $\gamma_{1}$ is determined by the antisymmetric part of viscous stress tensor $\sigma_{\mathrm{ij}}^{\prime}$, which cannot be proportional to the linear power of the symmetric tensor nematic order parameter $Q_{\mathrm{ij}}$, but at least its square. However, there is a delicate problem arising in the analysis of soft elasticity. The renormalised shear modulus $C_{5}^{R}=C_{5}-D_{2}^{2} / 8 D_{1}$ should reduce to the bare $C_{5} \rightarrow \frac{1}{2} \mu$ in the isotropic phase, at $Q \rightarrow 0$. However, the ideal values of $D_{1}$ and $D_{2}$ result in a finite renormalisation in this limit. One must revise the conclusions of non-ideal, semi-soft theory [11. Eq. (30) and the discussion in the Appendix suggest that, in fact, $D_{1}$ has a additional small (semi-soft) correction $\sim Q$. This resolves the problem of making the renormalisation $D_{2}^{2} / 8 D_{1}$ vanish at $Q \rightarrow 0$, but raises a question about the symmetry consideration that $\gamma_{1}$ (and $D_{1}$, by parallel) cannot be linear functions of $Q_{\mathrm{ij}}$. The paradox is safely resolved when one recognises that the semi-soft coefficient $a_{1}$ in eq. (30) is a linear function of $Q_{f}$, the order at network formation. Therefore, in fact, both $D_{1}$ and $\gamma_{1}$ in nematic elastomers depend on the bilinear combination $Q_{\mathrm{ij}}^{\{f\}} Q_{\mathrm{ij}}$, which means $\sim|Q|$, and thus no symmetry problem arises. In an ordinary liquid nematic there is no issue of formation order being frozen by crosslinking, and $\gamma_{1} \sim Q^{2}$ as expected.

\subsection{Balance of forces and torques}

To complete the general analysis of this Section, we briefly discuss the stresses and torques that follow from the combination of elastic and dissipation functions. In an overdamped system, traditionally ignoring inertial effects at low-frequency, we have two equations of motion: the balance of forces and torques. The first condition requires locally balancing the total symmetric stress tensor

$$
\underline{\underline{\boldsymbol{\sigma}}}=\underline{\underline{\boldsymbol{\sigma}}}_{\mathrm{el}}+\underline{\underline{\boldsymbol{\sigma}}}_{\mathrm{visc}}=\frac{\partial F}{\partial \underline{\underline{\varepsilon}}}+\frac{\partial(T \dot{s})}{\partial \underline{\underline{\dot{\varepsilon}}}}
$$

In the case when the relaxation time scales are separated, as in the discussion above, the viscous stress contribution to the force balance is minor. In contrast, the balance of torques, $\boldsymbol{\Gamma}=[\boldsymbol{n} \times \boldsymbol{h}]=0$ in the absence of external fields, requires the full molecular field

$$
\begin{aligned}
\boldsymbol{h}=\boldsymbol{h}_{\mathrm{el}}+\boldsymbol{h}_{\mathrm{visc}}= & D_{1}[\boldsymbol{n} \times(\boldsymbol{\Omega}-\boldsymbol{\omega})]+D_{2} \boldsymbol{n} \cdot \underline{\underline{\varepsilon}}+ \\
& +\gamma_{1}\left[\boldsymbol{n} \times \frac{d}{d t}(\boldsymbol{\Omega}-\boldsymbol{\omega})\right]+\gamma_{2} \boldsymbol{n} \cdot \underline{\underline{\dot{\varepsilon}}}
\end{aligned}
$$

Here both groups of terms are manifestly of the same order of magnitude in the regime of frequencies characterising director rotations. We shall see in the particular calculation of Section 4 that the condition of zero local torque allows one to obtain, for instance, the rate of director variation $\dot{\theta}$.

In the limit of isotropic rubber $Q \rightarrow 0$ the only relevant equation is that for the stress, which in this case reduces to

$$
\underline{\underline{\sigma}}=C_{4} \underline{\underline{\varepsilon}}+A_{4} \underline{\underline{\varepsilon}}
$$

which is a simplest viscoelastic approximation at lowest frequencies, or shear rates, of a general linear-response expression $\sigma(t)=\int G\left(t-t^{\prime}\right) \dot{\varepsilon}\left(t^{\prime}\right) d t^{\prime}$. Another known limiting case is that of an uncrosslinked nematic liquid crystal. In this case all equilibrium (zero-frequency) elastic moduli $C_{\mathrm{i}}$ and $D_{\mathrm{i}}$ are zero and the only contribution to the stress and torque balance are the Leslie-Ericksen eqs. (11). In a low molecular weight nematic, this is the full description of an anisotropic Newtonian liquid. In a polymer nematic, again, one expects a complex viscoelastic response function, $G\left(t-t^{\prime}\right)$, with several characteristic time scales, from the shortest Rouse time, to the entanglement and diffusion times (if applicable) [21]. Equations (11) are thus the low-frequency limit of such complex nematic viscoelasticity.

The theory of elastomer nematohydrodynamics has 5 phenomenological viscous coefficients. In a simplified singleviscosity model [7] we have found remarkable effects the internal director relaxation has on the macroscopic dynamicmechanical response of a nematic rubber. Here we follow the example of ordinary liquid crystals, where both the simplified and the full Leslie-Ericksen formalism have been used successfully over the years. In the practical calculation implementing the above ideas, we now move from the one-constant analysis of [7] to a full analysis.

\section{Shear stress and its relaxation}

\subsection{Simple shear deformations}

In a study of linear response, we shall first examine three principal simple shear geometries, as shown in Fig. 2. These are also the geometries that one achieves in a typical dynamic-mechanical experiment [7]. A geometry of uniaxial extension, more commonly found in studies of equilibrium stress-strain in elastomers, is less appropriate for an oscillating regime because of possible slow relaxation [26] and incomplete sample recovery on each cycle. The simple shear $\varepsilon(t)$, assumed externally applied to the sample (Fig. 2), is the single $x z$-component of the full Cauchy strain, the same for each director setup $\boldsymbol{G}, \boldsymbol{D}$ and $\boldsymbol{V}$. It 

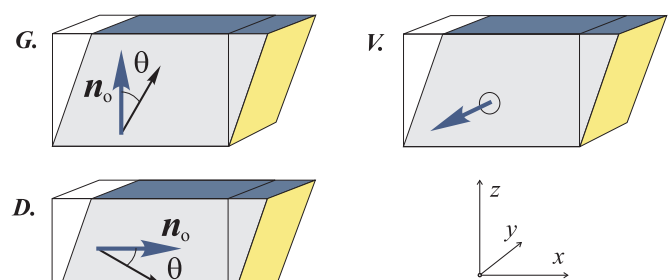

Fig. 2. The geometry of simple shear experiment with three principal orientations of the nematic director $\boldsymbol{n}_{0}$, labelled $G$ (for $\boldsymbol{n}_{0}$ along the shear gradient), $D$ (displacement) and $V$ (vorticity). The small-amplitude shear $\varepsilon_{x z} \sim \frac{1}{2} \varepsilon e^{\mathrm{i} \omega t}$ is applied to the elastomer and the measured stress $\sigma(\omega)$ provides the linear response modulus in each of the three configurations.

also automatically satisfies the necessary incompressibility constraint. We have then the symmetric part $\varepsilon_{z x}^{(s)}=\frac{1}{2} \varepsilon$ and the antisymmetric (body rotation) part expressed by $\Omega_{y}=\frac{1}{2} \varepsilon$.

Note that the three principal shear geometries in Fig. 2 are the same as in the classical setting for Miesovicz viscosity experiments. That is, if the director is kept immobile (e.g. by a strong external field, which incidentally would be hardly possible in elastomeric network under strain), then the orientations $\boldsymbol{V}, \boldsymbol{D}$ and $\boldsymbol{G}$ correspond to the Miesovicz viscosities $\eta_{\mathrm{a}}, \eta_{\mathrm{b}}$ and $\eta_{\mathrm{c}}$, respectively. Of course, the whole point of the present paper is to examine the effect the director freedom to rotate is having on the viscoelastic response.

We have argued above that Frank effects are subordinate in the overall balance of torques. Perhaps, this approximation needs to be reconsidered if a sheared sample is very thin in the $z$-direction: if the director is anchored on top and bottom surfaces (which are the plates in a shear-sandwich experiment), one expects a Frank energy density of the order $K / d^{2}$. The elastic energy density is set by $\mu$ and the two scales take on equal importance when $d=\xi=\sqrt{K / \mu}$, the nematic penetration depth. Taking typical values $K \sim 10^{-11} \mathrm{~J} / \mathrm{m}^{3}$ and $\mu \sim 10^{5} \mathrm{~J} / \mathrm{m}^{3}$ one has $\xi \sim 10^{-8} \mathrm{~m}$. Thus in practical situations with a sheared sample thickness $d \sim 100 \mu \mathrm{m}$ and more, ignoring the effect nematic director gradients seems to be a safe first approximation.

The remaining free energy density, eq. (2), takes the form, in the three cases of Fig. 22:

$$
\begin{aligned}
& F_{\mathrm{G}}=\left(C_{5}+\frac{1}{8}\left[D_{1}-2 D_{2}\right]\right) \varepsilon^{2}-\frac{1}{2}\left(D_{1}-D_{2}\right) \varepsilon \theta+\frac{1}{2} D_{1} \theta^{2} \\
& F_{\mathrm{D}}=\left(C_{5}+\frac{1}{8}\left[D_{1}+2 D_{2}\right]\right) \varepsilon^{2}-\frac{1}{2}\left(D_{1}+D_{2}\right) \varepsilon \theta+\frac{1}{2} D_{1} \theta^{2} \\
& F_{\mathrm{V}}=C_{4} \varepsilon^{2}
\end{aligned}
$$

where the small change in director orientation, $\delta \boldsymbol{n}$, is taken equal to the angle $\theta$. Clearly, one does not expect director rotation to occur in the "log-rolling" geometry $\boldsymbol{V}$.

The bilinear term $\sim \varepsilon \theta$ clearly reduces the energy: if a strain $\varepsilon$ is imposed then the director responds by adjusting $\theta$ to lower energy. Given sufficient time to reach equilibrium, $\theta$ adopts its optimal value for a given deformation $\varepsilon$. Returning this minimised value $\theta_{\mathrm{G}, \mathrm{D}}$ in 18 to
(17), the free energy at a given strain is also optimal:

$$
\theta_{\mathrm{G}, \mathrm{D}}=\frac{D_{1} \mp D_{2}}{2 D_{1}} \varepsilon, \quad F_{\mathrm{G}, \mathrm{D}} \rightarrow\left(C_{5}-\frac{D_{2}^{2}}{8 D_{1}}\right) \varepsilon^{2}
$$

Notice that if the nematic elastomer is ideal, then the free energies in the cases $\boldsymbol{G}$ and $\boldsymbol{D}$ vanish $\left(C_{5}-D_{2}^{2} / 8 D_{1}=0\right)$ because their geometry allows the director to respond to the shear and internally relax. Case $\boldsymbol{V}$ remains elastically hard. In fact, because of the chosen restricted strain geometry, Fig. 2, the response of even ideal elastomers is actually quartic, rather than completely soft (true softness requires some unconstrained extension as well as shear). The molecular model 11, 22 yields the quartic penalty $F=\frac{1}{2} \mu \frac{r^{2}}{(r-1)^{2}} \varepsilon^{4}$, which we are neglecting in the present linear-response analysis.

The generalised force driving the director rotation angle $\theta(t)$ to equilibrium is in fact a torque, $\partial F / \partial \theta$. In $F$ it is the externally imposed shear $\varepsilon(t)$, with its time variation, that is the ultimate driving agent. The flatness (softness) of the free energy $F(\varepsilon)$ will make the resulting dynamical response characteristically slow in the geometries where director rotation is possible [27]. Alternatively, if the imposed dynamics, e.g. $\varepsilon(t)$, is fast compared with that of $\theta(t)$, then one will not attain the ideal states (18) and the moduli will become frequency-dependent and deviate, for dynamic reasons, away from softness. It is this rheological subtlety that we now wish to examine.

\subsection{Viscoelastic response}

Driving a nematic elastomer by imposing a component of strain, in general, leaves open the possibility of dynamical response of not only the director but also the other components of strain (and thus involving for instance the translational viscosities $A_{1}, A_{5}$ and $\left.A_{5}\right)$. Then there will be several coupled dynamical equations for the system, see for instance [27] where the response of a nematic elastomer to a step-extensional strain is calculated. Here we confine ourselves to the dynamical response to imposed simple shear with other components of strain clamped. An elastic torque acting on nematic director $\boldsymbol{n}$ in certain deformation geometries is resisted by a linear viscous torque. Describing the director rotation by a small angle $\theta$, cf. Fig. 2, and continuing to ignore inertial effects, the balance of torques and forces is expressed by

$$
\frac{\delta F}{\delta \theta}+\frac{\delta(T \dot{s})}{\delta \dot{\theta}}=0
$$

The dynamical equation describing the evolution of the director approaching its equilibrium is given, for the two geometries where director rotation is present:

$$
\begin{aligned}
& (\boldsymbol{G}:) \quad \gamma_{1} \dot{\theta}=-D_{1} \theta+\frac{1}{2}\left(D_{1}-D_{2}\right) \varepsilon(t) \\
& +\frac{1}{2}\left(\gamma_{1}-\gamma_{2}\right) \dot{\varepsilon}(t) \\
& (\boldsymbol{D}:) \quad \gamma_{1} \dot{\theta}=-D_{1} \theta+\frac{1}{2}\left(D_{1}+D_{2}\right) \varepsilon(t) \\
& +\frac{1}{2}\left(\gamma_{1}+\gamma_{2}\right) \dot{\varepsilon}(t) \text {. }
\end{aligned}
$$


These linear, inhomogeneous differential equations are easily solved. After the transient relaxation $\theta \simeq \theta_{0} e^{-\left(D_{1} / \gamma_{1}\right) t}$ associated with starting the strain oscillations has completely relaxed, the steady-state response is given by the particular solutions with $\varepsilon \sim e^{\mathrm{i} \omega t}$ :

$$
\begin{aligned}
\theta_{\mathrm{G}, \mathrm{D}}(t)= & \int_{-\infty}^{t} d t^{\prime} e^{-\left(D_{1} / \gamma_{1}\left[t-t^{\prime}\right]\right)} \\
& \times\left(\frac{D_{1} \mp D_{2}}{2 \gamma_{1}} \varepsilon\left(t^{\prime}\right)+\frac{1}{2}\left(1 \mp \gamma_{2} / \gamma_{1}\right) \dot{\varepsilon}\left(t^{\prime}\right)\right)
\end{aligned}
$$

where the signs - or + correspond to the $\boldsymbol{G}$ or $\boldsymbol{D}$ geometry, respectively. The solutions depend on a characteristic time for director relaxation, $\tau_{1}=\gamma_{1} / D_{1}$, or equivalently, the characteristic frequency of the response scales like $D_{1} / \gamma_{1}$. We can now give a more concrete expression for the new dimensionless number $\mathrm{Ne}=\gamma_{1} \nabla v /\left(D_{1} \theta\right)$, since $\theta \sim \varepsilon$ and $\nabla v \sim \dot{\varepsilon} \sim \omega \varepsilon$, whence:

$$
\mathrm{Ne}=\gamma_{1} \omega / D_{1}=\tau_{1} \omega
$$

Below we shall see that all depends on $\tau_{1} \omega$ - when it is small, we have soft or semi-soft equilibrium elastic response, when it is large, we have hardening because director relaxation does not keep pace with changing strain.

The linear "nominal stress" in response to the imposed simple shear deformation $\varepsilon(t)$ is given by the sum of elastic and viscous stress functions $\sigma=\sigma_{\mathrm{el}}+\sigma_{\text {visc }}^{\prime}$, that is

$$
\sigma=\frac{\partial F}{\partial \varepsilon}+\frac{\partial(T \dot{s})}{\partial \dot{\varepsilon}}
$$

In the principal shear geometries, the stress expressions at a given frequency of imposed strain take the form

$$
\begin{aligned}
\sigma_{\mathrm{G}}(\omega)= & 2\left(C_{5}+\frac{1}{8}\left[D_{1}-2 D_{2}\right]\right) \varepsilon(\omega)-\frac{1}{2}\left(D_{1}-D_{2}\right) \theta(\omega) \\
& +2\left(A_{5}+\frac{1}{8}\left[\gamma_{1}-2 \gamma_{2}\right]\right) \dot{\varepsilon}-\frac{1}{2}\left(\gamma_{1}-\gamma_{2}\right) \dot{\theta} \\
\sigma_{\mathrm{D}}(\omega)= & 2\left(C_{5}+\frac{1}{8}\left[D_{1}+2 D_{2}\right]\right) \varepsilon(\omega)-\frac{1}{2}\left(D_{1}+D_{2}\right) \theta(\omega) \\
& +2\left(A_{5}+\frac{1}{8}\left[\gamma_{1}+2 \gamma_{2}\right]\right) \dot{\varepsilon}-\frac{1}{2}\left(\gamma_{1}+\gamma_{2}\right) \dot{\theta} \\
\sigma_{\mathrm{V}}(\omega)= & 2 C_{4} \varepsilon(\omega)+2 A_{4} \dot{\varepsilon} .
\end{aligned}
$$

One finds two kinds of viscous stress terms, of different orders of magnitude. The contribution $\gamma \dot{\theta} \sim \gamma \dot{\varepsilon}$ is of the same order as the elastic terms, as indicated by the torque balance eq. (20). In contrast, the terms $\sim A_{4,5} \dot{\varepsilon}$ are of the order $A \dot{\varepsilon} \sim C\left(\omega \tau_{\mathrm{R}}\right) \varepsilon \ll C \varepsilon$ at frequencies below Rouse values. The linear viscoelastic theory we are considering is applicable at much lower frequencies, where the most interesting physics is due to director relaxation and mechanical softness.

The effective response modulus in the "log-rolling" geometry $\boldsymbol{V}$ is unchanged by the nematic director dynamics, $G(\omega)=2 C_{4}+2 \mathrm{i} \omega A_{4}$ (the loss modulus negligible, as discussed above). In two other geometries, where the director rotation does take place, the dynamic modulus is modified by the internal director relaxation. Substituting the Fourier transforms of equations (21) into the expressions (24) and (25), we obtain the nominal stress in the form $\sigma(\omega)=G(\omega) \varepsilon(\omega)$. Remarkably, although perhaps
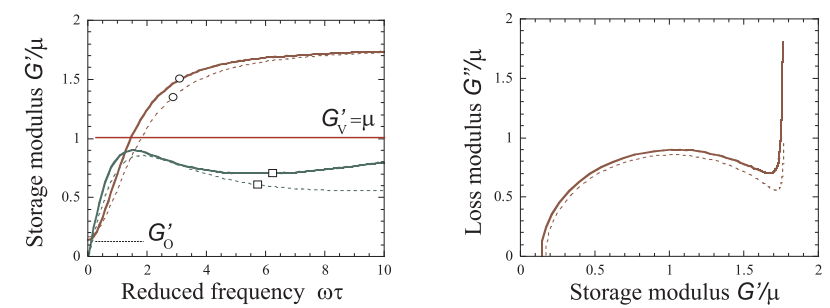

(a)

(b)

Fig. 3. (a) The frequency dependence of storage ( $G^{\prime}$, circles) and loss $\left(G^{\prime \prime}\right.$, squares) moduli in the two shear geometries $\boldsymbol{G}$ and $\boldsymbol{D}$ in units of rubber modulus $\mu$. There is no loss in the $\boldsymbol{V}$ geometry and, since $G_{\mathrm{V}}^{\prime}=\mu$, the plot represents the variation of the ratios $G_{\mathrm{G}}^{\prime} / G_{\mathrm{V}}^{\prime}$ and $G_{\mathrm{D}}^{\prime} / G_{\mathrm{V}}^{\prime}$. At $\omega \rightarrow 0$ both $G^{\prime} \rightarrow 2 C_{5}^{R}$. (b) The Cole-Cole plot, a variation of $G^{\prime \prime}$ with $G^{\prime}$. A semicircular shape indicates a single relaxation time response. In both plots, solid lines are for $Q=0.58$, dashed lines for $Q=0.35$.

predictably, the response in these two geometries is exactly the same: $\sigma_{\mathrm{G}}=\sigma_{\mathrm{D}}$, despite the difference in the rotations $\theta_{\mathrm{G}}$ and $\theta_{\mathrm{D}}$. The corresponding storage and loss moduli are given by the real and imaginary parts of the effective complex modulus $G(\omega)$. It has a single-relaxation time behaviour with a characteristic frequency $\omega_{1}=D_{1} / \gamma_{1}$ or the corresponding relaxation time $\tau_{1}=1 / \omega_{1}$. The dynamic moduli, in both $\boldsymbol{G}$ and $\boldsymbol{D}$ geometries, can then be written in a universal form:

$$
\begin{aligned}
G^{\prime}(\omega)= & 2\left(C_{5}-D_{2}^{2} / 8 D_{1}\right) \\
& +\frac{\left(\omega \tau_{1}\right)^{2}}{1+\left(\omega \tau_{1}\right)^{2}} \frac{\left(D_{2} \gamma_{1}-D_{1} \gamma_{2}\right)^{2}}{4 D_{1} \gamma_{1}^{2}} \\
G^{\prime \prime}(\omega)= & \frac{\omega \tau_{1}}{1+\left(\omega \tau_{1}\right)^{2}} \frac{\left(D_{2} \gamma_{1}-D_{1} \gamma_{2}\right)^{2}}{4 D_{1} \gamma_{1}^{2}} \\
+ & \omega \tau_{1}\left(\frac{1}{2} D_{1}\left(\gamma_{2} / \gamma_{1}\right)^{2}-2 A_{5} / \tau_{1}\right) .
\end{aligned}
$$

Figure 3 shows the example of frequency dependence of eqs. (27) and (28). In order to plot these functions, we need to make several numerical assumptions about the parameters. We thus take, arbitrarily for illustration purposes, $r=1+1.5 Q$ at a fixed temperature with values of the nematic order parameter corresponding to deep in a nematic phase, $Q=0.58$ (so that the chains are weakly prolate, $r \approx 1.87$ ), or corresponding to near a transition point, $Q=0.35$. The ratio $\gamma_{2} / \gamma_{1} \approx 1$ and the viscous constant $A_{5} \approx 0.1 \tau_{1} C_{5}$. Finally, the semi-soft addition to the coupling constant $D_{1}$ (see the Appendix) is taken as $a_{1}=0.1$. For these values, plots of $G^{\prime}$ and $G^{\prime \prime}$ reveal the expected single-relaxation behaviour. At zero frequency of imposed strain oscillations the real (storage) modulus in both shear geometries $\boldsymbol{G}$ and $\boldsymbol{D}$ is equal to

$$
G_{\mathrm{o}}^{\prime} \equiv 2 C_{5}^{\mathrm{R}}=2\left(C_{5}-D_{2}^{2} / 8 D_{1}\right)
$$

The measure of non-vanishing renormalised shear modulus $C_{5}^{\mathrm{R}} \neq 0$ is the characteristic parameter of semi-softness in non-ideal nematic elastomers. The high frequency response for $\omega \tau_{1} \gg 1$ is

$$
G_{\infty}^{\prime}=2 C_{5}-\frac{1}{2} D_{2}\left(\gamma_{2} / \gamma_{1}\right)+\frac{1}{4} D_{1}\left(\gamma_{2} / \gamma_{1}\right)^{2}
$$


The characteristic single-relaxation time behaviour seen in fig. 3 is due to our assumption about separation of time scales, $\tau_{1} \gg \tau_{\mathrm{R}}$. For that reason, we are able to distinguish the plateau in $G^{\prime}$, the maximum and the "high-frequency" drop in $G^{\prime \prime}$ and a semicircular shape of Cole-Cole plot [28]. For the chosen value of $A_{5}$, one can begin to see the next rise in $G^{\prime \prime}$, when the frequency approaches the next characteristic point, the Rouse frequency, leading towards the glass plateau value $G^{\prime} \sim 10^{11} \mathrm{~Pa}$ at high frequency. That transition would correspond to another semi-circular Cole-Cole plot at much higher values of $G^{\prime}$; we can see the beginning of that graph segment in fig. 3(b). With the assumed separation of nematic and rubber relaxation time scales (and the assumed ideal polymer network, with no entanglements and free dangling ends, which are known to lead to slow relaxation), the mechanical losses at low frequencies are only through the lag in director rotation.

The initial expressions, eqs. (24) and (25), differ in sign of terms with $D_{2}$ and $\gamma_{2}$ constants. This reflects the tendency to align or repel the director by these terms, the roles being reversed when we interchange prolate and oblate symmetry $\left(r>1\right.$ and $r<1$, the sign of both $D_{2}$ and $\gamma_{2}$ reversing on this exchange). However, when the director relaxation (21) is substituted back into the stress expressions, one only finds the products $D_{2} \gamma_{2}$, and a corresponding unique effective complex modulus. Another feature of $G^{*}(\omega)$ is the non-dimensional ratio $\gamma_{2} / \gamma_{1}$, which is a familiar parameter in the dynamics of ordinary liquid crystals [8] in the context of director flow alignment or tumbling. As the brief discussion of modified torque balance in Section 3 indicates, one does not expect a steadystate tumbling in elastomers, where there is memory of the original director through the elastic energy. Tumbling and analogous effects may only be found in transient regimes or in non-linear elasticity, to which we return elsewhere.

Variation of dynamic complex moduli with temperature is very interesting to examine graphically, as well as analytically. Figure 1(a) shows the dependence of storage modulus $G^{\prime}$ on the reduced temperature $T / T_{\mathrm{ni}}$, for a sequence of increasing frequency values. In order to produce these plots, we must take a further assumption about the variation of the order parameter $Q(T)$. We take, rather arbitrarily, $Q \approx\left(1-T / T_{\mathrm{ni}}\right)^{0.33}$ simply because this was an approximate fit to the experimental measurement of $Q(T)$ in [7]. We also take, following classical nematics, $\gamma_{1}=g_{1} Q^{2}$ and $\gamma_{2}=g_{2} Q$ and further fix $g_{2} / g_{1}=\frac{1}{2}$ to have $\gamma_{2} \approx \gamma_{1}$ at $Q \sim 0.5$. These rather limiting assumptions nevertheless usefully illustrate the qualitative behaviour of the linear elastic response functions both deep in the nematic phase and near the assumed "critical point" $T_{\mathrm{ni}}$.

The characteristic time of director relaxation $\tau_{1}=$ $\gamma_{1} / D_{1}$ is a function of temperature through its dependence on nematic order parameter. Both the coupling constant $D_{1}$ and the rotational viscous coefficient $\gamma_{1}$ are functions of $Q(T)$, as discussed in Section 3. Hence the relaxation time $\tau_{1}$ should have a weak $Q$-dependence in the nematic phase, which should change to $\tau_{1} \sim|Q|$ in the vicinity of the nematic-isotropic transition point. We discuss subtle limit problems and the form of $G(T, \omega)$ as a function of $T$
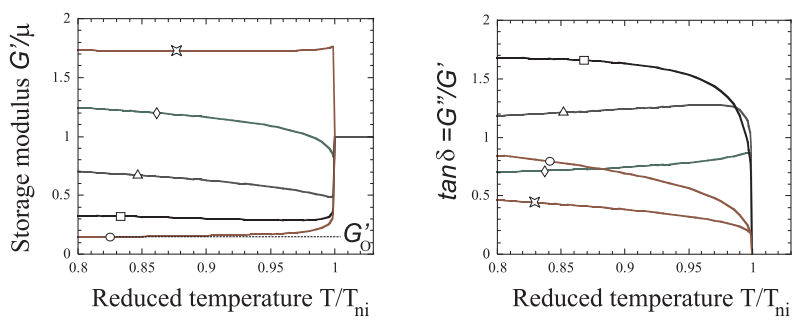

(a)

Fig. 4. (a) Plot of reduced storage moduli $G^{\prime} / \mu$ against reduced temperature for a number of increasing reduced frequencies: $\tilde{\omega}=0.1$ (circles), 0.5 (squares), 1 (triangles), 2 (diamonds) and 10 (stars). Moduli approach the semi-soft constant $G_{\mathrm{o}}^{\prime}$ at zero frequency and low temperature, cf. Fig. B(a). An apparent critical behaviour near the transition point $T_{\mathrm{ni}}$ is due to the nematic order parameter variation $Q(T)$ appearing in the elastic and viscous coefficients. (b) Plot of loss factor $\tan \delta=G^{\prime \prime} / G^{\prime}$ against reduced temperature for a number of increasing frequencies. The curves are labeled the same as in (a).

for various fixed $\omega$ in the Appendix. The combination $\omega \tau_{1}$ can be rewritten as

$$
\omega \tau_{1} \equiv \omega \frac{\gamma_{1}}{D_{1}}=\omega\left(\frac{g_{1}}{\mu}\right) \frac{Q^{2}}{a_{1}|Q|+(r-1)^{2} / r},
$$

where we recall that $r \sim 1+\beta Q$ (in practice, the linear relation holds to high values of $Q$ [7, 31]). We shall continue taking $\beta=1.5$ in our illustration. One can also scale $\omega$ by $\mu / g_{1}$ to give a dimensionless frequency $\tilde{\omega}$; thus $\omega \tau_{1}=$ $\tilde{\omega} f(Q)$ where the non-dimensional function $f(Q)$ is clearly $f \sim|Q|$ as $Q \rightarrow 0$.

The cusps near $T_{\text {ni }}$ seen in Fig. 4 (a) are a consequence of our assumption of the critical form $Q(T) \sim\left(1-T / T_{\mathrm{ni}}\right)^{\cdot 33}$. For a real, non-ideal monodomain nematic elastomer with of necessity a high-temperature paranematic state and a residual small $Q$ above $T_{\text {ni }}$, the cusps are rounded off but the general form survives, including the non-monotonic variation $G^{\prime}(T)$ at high frequencies. We note in passing, that the experimental result for $G^{\prime}(T)[7]$ is very close to these predictions: although the very high frequency was not achieved, the gradual departure of $G^{\prime}(T) / \mu$ from the universal low-frequency curve was clearly registered.

Figure 1(b) also shows the unusually high loss tangent arising from the real and imaginary parts of complex modulus. The new loss mechanism, due to internal director relaxation, is additional to all classical losses occurring in polymer systems - in particular those occurring near the glass transition. The information about the latter is, as before, contained in the essential frequency and temperature dependence of rubber-elastic constant $\mu$, of which the expressions for $F$ and $T \dot{s}$ are the low-frequency limit. There is a striking contrast between the fig. A(b) with unusually high values of $\tan \delta$ over a broad temperature range and the traditional distinct loss peaks in classical polymers and rubbers. Physically, the maximal loss occurs where the the imposed strain frequency, $\omega$, matches the director relaxation rate $1 / \tau_{1}$. We have a crossover from the director keeping up with strain and therefore allowing the the system to be very soft, to where the director fails to re- 
spond quickly enough and the system becomes hard. The large change of modulus when one crosses this frequency interval is manifested, by the Kramers-Krönig relations, in a very high loss peak.

\section{Conclusions}

We have developed a model of time-dependent response and rheology of nematic elastomers by coupling nematic rubber elasticity with nematohydrodynamics. The theory represents the low-frequency limit of general viscoelastic description of nematic polymer networks (which is not known). By making use of significant separation between the natural nematic relaxation time scale and that of a rubber network, we examine the dissipation at relatively low frequencies due to internal director relaxation in a deformed elastomer. Because of the lagging of director rotation in response to time-dependent imposed shears, soft or semi-soft response can only be partially achieved, the hardening depending on frequency and on temperature. The nemato-elastic-hydrodynamic equations are derived in generality and solved for the rheology of a particular, important example, that of simple oscillating shear, giving rise to unusual temperature dependence of response moduli and loss tangents $(\tan \delta \sim 1.5-2)$. Thus these results will be important for a wide variety of applications in damping, acoustics, frequency and directional dependent materials, and so on.

We have already reported on rheological experiments [7] that show the qualitative features of our theory. These and light scattering experiments 29,30 give values of Frank constants, rubber constants $C_{5}, D_{1}, D_{2}$ and the rotational viscosity coefficient $\gamma_{1}$ for nematic elastomers (and the $\gamma_{1}$ also for the corresponding polymer melts). The $C$ 's and $D$ 's can also be deduced from several static measurements, in all cases giving typical rubber values $\sim 10^{3}-10^{5} \mathrm{~Pa}$, modulated by the discussed $Q$-dependence. Frank constants take values typical of classical nematic liquids, $K \sim 10^{-11} \mathrm{~N}$. But the $\gamma$ values are enhanced by polymerisation. This is not surprising since on reorienting a chain one must translate it bodily. An extended object sweeps through space and the dynamics are complex. In a network this is even less straightforward because the chain is mechanically tied to a matrix and must resolve its motions with those of other chains. It turns out that the $\gamma$ 's are further enhanced, reaching values of order $\sim 10^{3} \mathrm{~Pa}$.s and more, leading to the mentioned separation of characteristic time scales.

We appreciate valuable discussions with S.M Clarke, H. Finkelmann, T.C. Lubensky, R.B. Meyer, P.D. Olmsted and W. Stille.

\section{A Semi-softness and rheology}

At fixed temperature in the nematic state, Fig. 3 shows the rheological consequences of nematohydrodynamics in elastomers - as frequency drops and director relaxation had keep pace with imposed strains, the response gets ever softer. In the limit $\omega \tau_{1} \rightarrow 0$ it either vanishes (ideal softness) or becomes very small (semi-softness) as has been confirmed by many quasi-static experiments. We wish to comment on the mechanisms for softness and non-ideality and then how, at various fixed frequencies, one can see how $G^{\prime}$ tends to conventional rubber elastic response as $T$ increases and $Q \rightarrow 0$.

Soft shear response occurs because anisotropic network chains accommodate a macroscopic shape change by rotating their distribution of shapes at constant entropy. When chains tend to isotropy, $Q \rightarrow 0$ and $r \rightarrow 1$, shape change can only be accommodated by chains distorting their distribution, thereby decreasing their entropy and increasing their free energy. The modulus is then $\mu$. We must see how this classical limit is achieved; after all the cancellation $C_{5}^{R}=C_{5}-D_{2}^{2} / 8 D_{1}=0$ appears to hold for all $r$ including $r \rightarrow 1$.

Semi-softness is expected when there are fluctuations of composition, rod-like crosslinks, sources of random nematic field, and any other form of non-ideality that prohibits the finding of an isotropic reference state 22, 23. Then there are additions to the Trace formula (11) which are of the form $a \sin ^{2} \theta$ for simple shears, see for instance [32. The degree of semi-softness, $a$, can be calculated directly from various models of non-ideality, or measured. For continuum theory such additions appear in the eqs. (3) for the $C_{i}$ and $D_{i}$, and upset the cancellations in (4), yielding a $C_{5}^{R} \neq 0$, which is the non zero limit at $\omega \tau_{1} \rightarrow 0$ in Fig. 3 for $G^{\prime}$. However, the additions inspired by $a$ clearly must vanish as $Q \rightarrow 0$ and $r \rightarrow 1$ and the delicate limit question must still be resolved. In particular in (仹) $C_{5}^{R} \rightarrow C_{5} \rightarrow \frac{1}{2} \mu$ as $r \rightarrow 1$.

In the ideal case there is no high temperature order, softness is perfect and for $T<T_{\mathrm{ni}}$ the modulus $C_{5}^{R}$ is identically zero. Several systems, depending on their thermomechanical history, are close to this 22 . Since ideally $Q$ jumps to zero at $T=T_{\mathrm{ni}}$, there is no limit problem. $D_{1}$ and $D_{2}$ cease to exist, the analysis (18) is invalid and $C_{5}$ is not renormalised.

In the semi-soft case, (4) places bounds on the form the non ideal additions to $D_{1}$ can take. Since $D_{1} \sim Q^{2}$ and $D_{2} \sim Q$, in the ideal case the limit $D_{2}^{2} / D_{1}$ is finite as $Q \rightarrow 0$. If however $D_{1}$ has additions $D_{1} \sim\left(Q^{2}+a_{1} Q\right)$, then the limit of $D_{2}^{2} / D_{1}$ vanishes as $Q \rightarrow 0$, eliminating any renormalisation. Explicit calculations agree with symmetry arguments that the additions to $D_{1}$ are indeed $\sim Q$ and reflect the thermomechanical history of the material. For instance fluctuations (due to compositional fluctuations in the polymers that make up the network) in the effective order felt by a chain, $Q_{\text {eff }}=(1+\delta) Q$ (with $\langle\delta\rangle=0$ ), yield for the modulus:

$$
D_{1}=\mu^{\prime}\left[\frac{(r-1)^{2}}{r}+a_{1} Q\right]
$$


where $\mu^{\prime}=\left(\frac{\operatorname{det} \underline{\underline{\ell}}_{f}}{\operatorname{det} \underline{\underline{\ell}}}\right) \mu$ and

$$
a_{1}=3\left\langle\delta^{2}\right\rangle \frac{Q_{f}\left(3+2 Q_{f}\right)}{\left(1+2 Q_{f}\right)\left(1-Q_{f}\right)} .
$$

Here formation conditions for the network are denoted by a subscript $f$ : the step length tensor at formation is $\underline{\underline{\ell}}_{f}$ with order parameter $Q_{f}$. The determinant-factors reflect spontaneous shape changes since formation which can be very large (up to several hundred \%). The sign of the coefficient $a_{1}$ is that of $Q_{f}$. It is the order at network formation that induces the residual order $Q$ at high temperatures and the sign of that order follows that of the formation order. Thus the combination $a_{1} Q$ is always positive and thus the non-ideal additions to $D_{1}$ in $(30)$ are really of the form $|Q|$, which we have used in section 3.4.

Thus at sufficiently low frequencies one observes a rather small modulus $G^{\prime}=C_{5}^{R} \geq 0$, which rises with increasing temperature to reach the classical value $\mu$ at $Q \rightarrow 0$ at $T_{\text {ni }}$. If, at fixed frequency, on dropping the temperature the combination $\omega \tau_{1}$ becomes appreciable (due to increasing $\tau_{1}$ ), the modulus $G^{\prime}(T)$ will depart from the universal low-frequency form $G_{\omega \tau_{1} \rightarrow 0}^{\prime}$, see Fig. 4 .

\section{References}

1. J.L. Gallani, L. Hilliou, P. Martinoty, F. Doublet and M. Mauzac, J. Phys. II France 6, 443 (1996).

2. J. Weilepp, P. Stein, N. Assfalg, H. Finkelmann, P. Martinoty and H.R. Brand, Europhys. Lett. 47, 508 (1999).

3. E.M. Terentjev and M. Warner, J. Phys. II France 4, 111 (1994).

4. J. Weilepp and H.R. Brand, Macromol. Theory Simul. 7, 91 (1998).

5. E. Nishikawa and H. Finkelmann, Macromol. Rapid Comm. 19, 181 (1998).

6. M.J. Osborne and E.M. Terentjev, Phys. Rev. E 62, 5101 (2000).

7. S.M. Clarke, A.R. Tajbakhsh, E.M. Terentjev and M. Warner, Phys. Rev. Letts. submitted

8. P.G. de Gennes and J. Prost, Physics of Liquid Crystals (Clarendon, Oxford, 1993).

9. T.C. Lubensky, Phys. Rev. A2, 2497 (1970).

10. E.M. Terentjev, J. Phys. Cond. Mat. 11, R239 (1999).

11. M. Warner and E.M. Terentjev, Progr. Polym. Sci. 21, 853 (1996).

12. P.D. Olmsted, J. Phys. II 4, 2215 (1994).

13. L.D. Landau and E.M. Lifshitz, Theory of elasticity, $3^{\mathrm{d}}$ ed. (Pergamon, Oxford, 1986).

14. P.G. de Gennes in: Liquid Crystals of One- and TwoDimensional Order, ed. W. Helfrich and G. Heppke, Springer, Berlin, 231 (1980).

15. M. Warner, E.M. Terentjev, R.B. Meyer and Y. Mao, Phys. Rev. Lett. 85, 2320 (2000).

16. H. Imura and K. Okano, Japan. J. Appl. Phys. 11, 1440 (1972).

17. N. Kuzuu and M. Doi, J. Phys. Soc. Japan, 52, 3486 (1983).

18. M.A. Osipov and E.M. Terentjev, Z. Naturforsch. 44a, 785 (1989).
19. W.H. de Jeu, Physical Properties of Liquid Crystalline Materials, Gordon and Breach, NY (1980).

20. S.F. Edwards, H. Takano and E.M. Terentjev, J. Chem. Phys. 113, 5531 (2000).

21. M. Doi and S.F. Edwards, Theory of Polymer Dynamics, Clarendon, Oxford (1986).

22. M. Warner, J. Mech. Phys. Solids 47, 1355 (1999).

23. L. Golubovic and T.C. Lubensky, Phys. Rev. Lett. 63, 1082 (1989).

24. G.E. Volovik, JETP Lett. 31, 273 (1980).

25. T. Carlsson, J. Physique, 44, 909 (1983).

26. S.M. Clarke and E.M. Terentjev, Phys. Rev. Lett. 81, 4436 (1998).

27. P.I.C. Teixeira and M. Warner, Phys. Rev. E60, 603 (1999).

28. J.D. Ferry, Viscoelastic Properties of Polymers (John Wiley \& sons, New York, 1980).

29. J. Schmidtke, W. Stille and G. Strobl, Macromolecules 33, 2922 (2000).

30. R.B. Meyer, unpublished light scattering results.

31. H. Finkelmann, A. Greve and M. Warner, Eur. Phys. J. E submitted (2000).

32. G.C. Verwey, M. Warner and E.M. Terentjev, J. Phys. II France, 6, 1273 (1996). 